\title{
Emotional cues enhance the attentional effects on spatial and temporal resolution
}

\author{
Bruno R. Bocanegra $\cdot$ René Zeelenberg
}

Published online: 7 September 2011

(C) The Author(s) 2011. This article is published with open access at Springerlink.com

\begin{abstract}
In the present study, we demonstrated that the emotional significance of a spatial cue enhances the effect of covert attention on spatial and temporal resolution (i.e., our ability to discriminate small spatial details and fast temporal flicker). Our results indicated that fearful face cues, as compared with neutral face cues, enhanced the attentional benefits in spatial resolution but also enhanced the attentional deficits in temporal resolution. Furthermore, we observed that the overall magnitudes of individuals' attentional effects correlated strongly with the magnitude of the emotion $\times$ attention interaction effect. Combined, these findings provide strong support for the idea that emotion enhances the strength of a cue's attentional response.
\end{abstract}

Keywords Emotion · Attention - Visual perception · Fearful faces $\cdot$ Spatiotemporal resolution

Attention can be allocated overtly, by directing one's gaze, or covertly, to a peripheral location without eye movements (Egeth \& Yantis, 1997). Covert attention has been shown to facilitate a variety of basic perceptual dimensions, such as contrast sensitivity and spatial resolution (Cameron, Tai, \& Carrasco, 2002; Carrasco \& Yeshurun, 2009; Yeshurun \& Carrasco, 1999), allowing a more sensitive, fine-grained perceptual analysis at the cued spatial location.

Recent findings indicate that emotion, independently of attention, also influences a variety of basic perceptual dimensions, such as contrast sensitivity, orientation sensitivity, and spatial resolution (Bocanegra \& Zeelenberg,

B. R. Bocanegra $(\bowtie) \cdot$ R. Zeelenberg

Department of Psychology, Erasmus University Rotterdam,

Woudestein, T13-26, P.O. Box 1738, 3000 DR Rotterdam,

The Netherlands

e-mail: bocanegra@fsw.eur.nl 2009b, 2011; Phelps, Ling, \& Carrasco, 2006). Interestingly, one of these studies has provided the first evidence that emotion and attention exert an interactive influence on perception. Phelps et al. (Experiment 2) presented fearful or neutral face cues prior to four peripheral Gabor patches presented at near-threshold contrast. One of the Gabors was a tilted target, and the others were upright distractors; the observers' task was to indicate the orientation of the target Gabor. To manipulate attention, either a single face cued the location of the upcoming target (a valid informative cue) or multiple faces cued all four Gabors (a distributed uninformative cue). A typical attentional effect was observed: Valid cues enhanced contrast sensitivity, as compared with distributed cues. Importantly, the effects of emotion and attention were superadditive: The beneficial effect of attention on contrast sensitivity (i.e., the difference in performance between valid and distributed cues) was larger for emotional cues than for neutral cues. ${ }^{1}$

To our knowledge, no study has yet extended this interaction to other basic perceptual dimensions. As a consequence, the mechanisms underlying emotionattention interactions in vision are still largely unspecified. In the present study, we tested whether emotion enhances

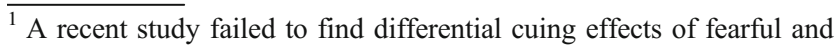
neutral faces (Ferneyhough, Stanley, Phelps, \& Carrasco, 2010). Here, the targets appeared below the location of the face cues, whereas in the present experiments and Phelps et al. (2006), they appeared alongside the faces around the nose-eyes region. Considering that this is the most diagnostically informative region for emotion discrimination (Smith, Cottrell, Gosselin, \& Schyns, 2005), it could be that face cuing effects diminish as targets are presented further away from this subregion. Alternatively, Ferneyhough et al., suggested that their null effect was due to the mid-level spatial frequency used to construct their targets $(4 \mathrm{cpd})$, considering that the beneficial effect of emotion on perception (independently of attention) is restricted to low spatial frequencies (Bocanegra \& Zeelenberg, 2009b).
} 
the effect of attention in two new visual dimensions: spatial and temporal resolution (Yeshurun \& Carrasco, 1999; Yeshurun \& Levy, 2003).

It has been proposed that valid emotional cues influence perception more strongly than do valid neutral cues, because they are more salient and attract additional attentional resources (Phelps et al., 2006; Bocanegra \& Zeelenberg, 2009a) emotion paper "dissociating emotion-induced blindness and hypervision." Analogously, bright attentional cues have a stronger effect on target perception than do faint attentional cues (Fuller, Park, \& Carrasco, 2009). According to this account, the interaction between emotion and attention reflects an increased attentional response at the cued location.

However, spatial cues not only allocate attention to a target's location, but also reduce the spatial uncertainty of the target (Luck \& Thomas, 1999). Although the higher salience of emotional cues could, indeed, enhance the attentional response at the cued location, the more salient valid emotional cues could also be more effective than valid neutral cues at restricting the perceptual decision to the information presented at the cued location. To illustrate, in the Phelps et al. (2006) study, the simultaneous presentation of near-threshold Gabors at all four locations could have made it difficult for observers to ascertain which location contained the target. Consequently, performance may also have been influenced by noise arising from the three distractor locations, in addition to the signal at the target location (Luck \& Thomas, 1999). Considering that on valid trials, the cue correctly predicted the location of the upcoming target, observers could have attained a higher level of accuracy on these trials by restricting their perceptual decision to information presented at the cued location and disregarding information at the uncued locations. In this manner, a reduction in spatial uncertainty of the target could have improved performance by diminishing the impact of external noise on the perceptual decision (Carrasco, Williams, \& Yeshurun, 2002).

In Experiment 1, we investigated the effect of attention and emotion on spatial resolution in a spatial gap detection task. Unlike in the Phelps et al. (2006) study, displays contained a single suprathreshold target whose high contrast eliminated the spatial uncertainty associated with near-threshold Gabors. In addition, the display lacked any sources of external noise, such as distractors or masks. An attentional account predicts that valid emotion cues should have a stronger beneficial effect on spatial resolution, as compared with valid neutral cues, in the absence of competing distractors. Because, in this task, the suprathreshold target could be not be confused with either distractors or empty locations, any effects of emotion and attention are unlikely to be due to a reduction in spatial uncertainty (Carrasco et al., 2002).

\section{Experiment 1}

We presented a single valid cue adjacent to the location of a subsequent target, in order to direct attention to that location. In a comparison condition, a distributed cue appeared at the two possible target locations and, thus, was uninformative as to the upcoming target's location (see Fig. 1). We expected to replicate previously observed attentional benefits in spatial resolution (Carrasco et al., 2002; Montagna, Pestilli, \& Carrasco, 2009; Yeshurun \& Carrasco, 1999). If the attentional response hypothesis is correct, emotional cues should enhance the beneficial effect of attention on spatial resolution in the absence of target spatial uncertainty.

Method

Participants Twenty-two observers with normal or corrected-to-normal vision participated in the experiment and gave informed consent before participation. All procedures were approved by the local ethics committee.

Stimuli and apparatus A white fixation point $\left(0.3^{\circ} \times\right.$ $0.3^{\circ}, 65 \mathrm{~cd} / \mathrm{m}^{2}$ ) was presented centrally in a uniform dark background $\left(2 \mathrm{~cd} / \mathrm{m}^{2}\right)$, throughout each trial. To manipulate emotion, 11 facial photographs portraying fearful and neutral expressions were selected from Ekman and Friesen (1976). Valid cues consisted of a single face stimulus (either fearful or neutral, $5^{\circ}$ in diameter) presented either left or right of fixation next to the target location at $8^{\circ}$ eccentricity. Distributed cues consisted of two identical face stimuli, both either fearful

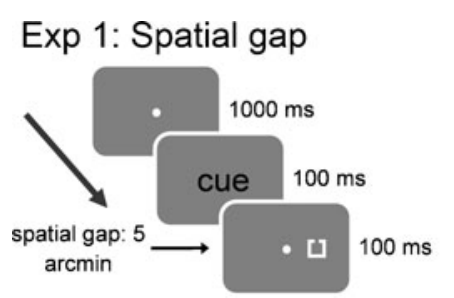

\section{Exp 2: Temporal gap}
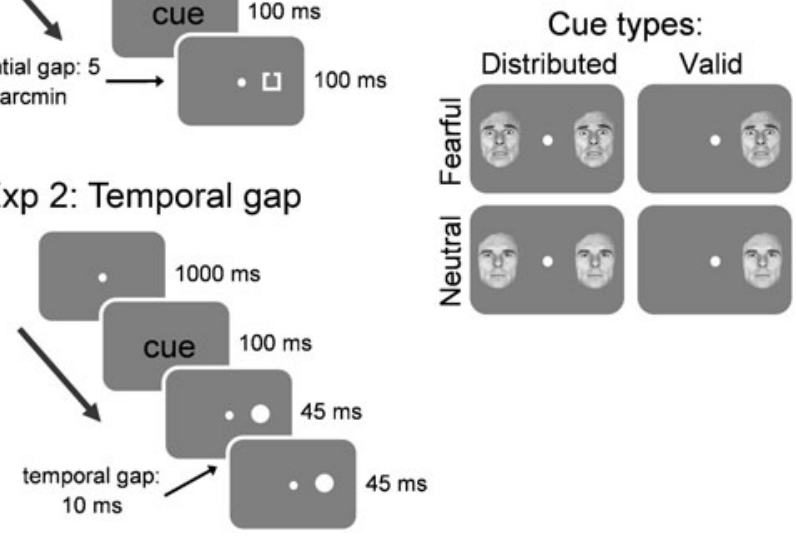

Fig. 1 Illustration of the display sequences for the trials in the experiments. Please note that the cue stimuli were presented until the offset of the target stimulus in both the spatial and temporal tasks 
or neutral, presented left and right of fixation next to the two possible target locations. ${ }^{2}$

The target was a Landolt square $\left(1^{\circ}, 65 \mathrm{~cd} / \mathrm{m}^{2}\right)$, randomly presented either left or right of fixation at $4^{\circ}$ eccentricity. The Landolt was presented for $100 \mathrm{~ms}$ and contained a very small aperture at the top ( 5 arcmin) on $50 \%$ of the trials and no aperture on the remaining trials. Stimuli were presented on a gamma-corrected Iiyama 21-in. Vision Master monitor (100-Hz refresh-rate, 1,600 × 1,200 pixels).

Procedure Observers viewed the display binocularly at a distance of approximately $60 \mathrm{~cm}$, fixating centrally throughout testing. First, the fixation was presented for $1,000 \mathrm{~ms}$, followed by the cue $(100 \mathrm{~ms})$ (see Fig. 1). Next, the target was added to the display. The short duration between cue onset and target offset $(200 \mathrm{~ms})$ precluded eye movements toward the stimuli (Mayfrank, Kimmig, \& Fischer, 1987). Observers indicated whether the Landolt square contained a small spatial gap at the top (by pressing the " $\mathrm{m}$ " key) or whether the square was intact (by pressing the " $\mathrm{z}$ " key). Each observer performed 100 training trials prior to the main experiment, which consisted of 880 trials. All variables varied randomly from trial to trial, and performance feedback was given after each trial.

\section{Results and discussion}

We analyzed our data using signal detection theory measure $d^{\prime}[z$ (hit) $-z$ (false alarm) $]$ and criterion $C[-.5 *(z$ (hit $)+z$ (false alarm))], whhich we calculated for each condition and observer (Macmillan \& Creelman, 1991). Three ANOVAs with attention (valid vs. distributed cue) and emotion (fearful vs. neutral cue) as within-subjects factors were performed on $d^{\prime}$ accuracy, criterion $C$, and response times (RTs) for correct responses, respectively.

The ANOVA on $d^{\prime}$ accuracies showed a significant main effect of attention, $F(1,21)=41.96, p<.001, \eta_{\mathrm{p}}{ }^{2}=.67$, indicating that valid cues improved performance, as compared with distributed cues (see Fig. 2). Also, fearful cues improved performance, as compared with neutral cues, $F(1,21)=6.49, p<.03, \eta_{\mathrm{p}}{ }^{2}=.24$. Most important, the effects of attention and emotion interacted, $F(1,21)=$ 13.34, $p<.01, \eta_{\mathrm{p}}{ }^{2}=.39$. As is illustrated in the upper part of Fig. 3, this interaction meant that the attentional benefit (i.e., the difference between valid and distributed cues) was

\footnotetext{
$\overline{2}$ Please note that target spatial variability in the experiments does not imply target spatial uncertainty. Observers were completely certain about where a target was presented (due to its high contrast and lack of distractors or masks), despite cross-trial variability in the target location.
}

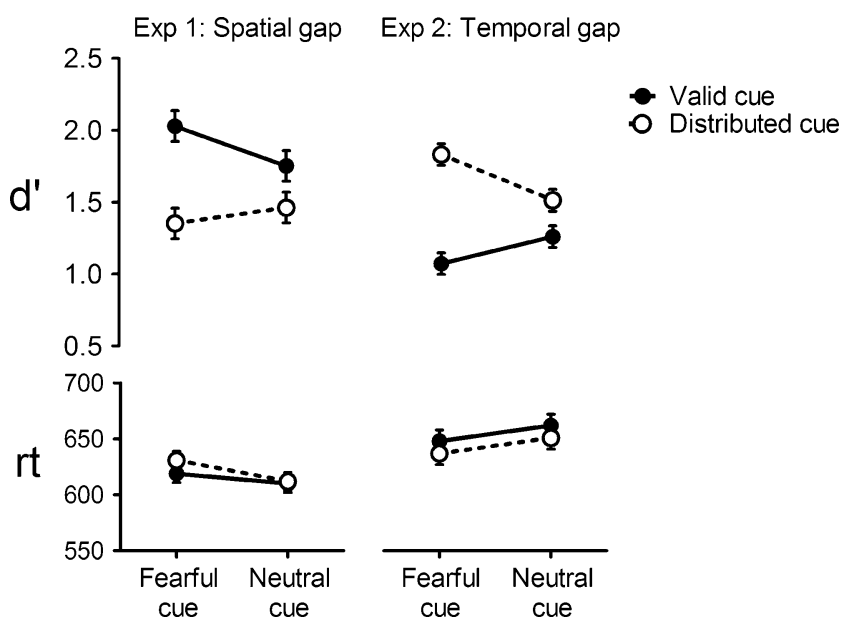

Fig. $2 d^{\prime}$ performance and RTs for each of the experimental conditions in the spatial and temporal resolution tasks. Error bars represent within-subjects standard errors of the means (Loftus \& Masson, 1994)

larger for fearful cues (0.68 $d^{\prime}$ difference) than for neutral cues (0.29 $d^{\prime}$ difference). Planned comparisons using the pooled variance estimate (Loftus \& Masson, 1994) indicated that the attention effect was significant for both fearful and neutral cues, $t(21)=6.09, p<.01$, and $t(21)=2.64, p<.02$, respectively. Also, fearful distributed cues, as compared with neutral distributed cues, impaired spatial resolution, $t(21)=2.19, p<.05$. The present results indicate that emotional cues enhance the beneficial effect of attention on spatial resolution in the absence of target spatial uncertainty, which is consistent with the attentional response hypothesis. Effects on criterion $C$ and RTs for the main effects and the interaction were not significant $(p s>.12)$.

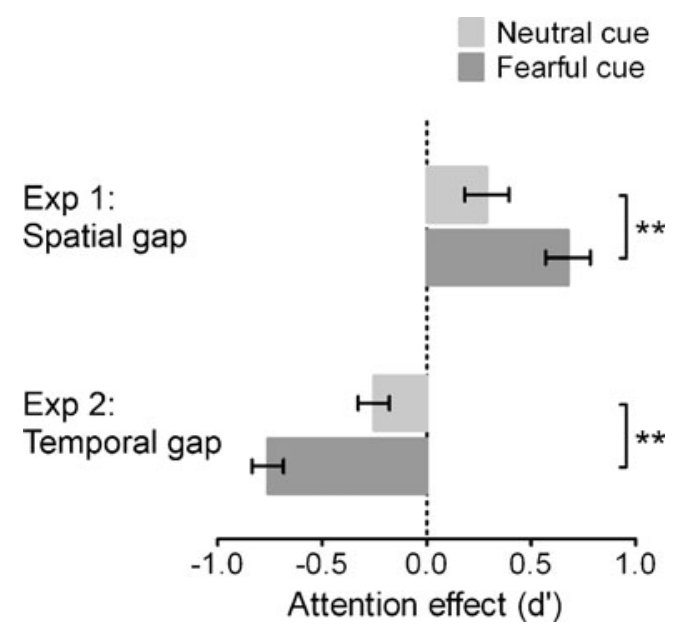

Fig. 3 Attention effects for neutral and fearful cues in the spatial and temporal resolution tasks. The attention effect was calculated by subtracting performance in the valid condition from performance in the distributed condition. Error bars represent within-subjects standard errors of the means (Loftus \& Masson, 1994) 


\section{Experiment 2}

In Experiment 2, we explored the effect of attention and emotion in a perceptual task in which performance would be impaired by attention allocation. Attention increases spatial resolution at the cued location, thereby improving our ability to resolve fine details (Yeshurun \& Carrasco, 1999). Counterintuitively, however, this spatial benefit is accompanied by a cost in temporal resolution: Attention allocation impairs our ability to resolve fast temporal changes in luminance (Rolke, Dinkelbach, Hein, \& Ulrich, 2008; Yeshurun \& Levy, 2003; but see Chica \& Christie, 2009).

Although counterintuitive, this temporal deficit is less surprising when one considers the beneficial effect of attention on spatial resolution (Yeshurun \& Carrasco, 1999). From the retinal ganglion level up to V1, the visual system consists of two parallel visual channels (Callaway, 1998), parvocellular and magnocellular. In general, parvocellular neurons have smaller receptive fields and more sustained responses, whereas magnocellular neurons have larger receptive fields and transient responses. It has been suggested that attention modulates inhibitory interactions between magnocellular and parvocellular channels (Yeshurun \& Levy, 2003). In this manner, attention transiently sacrifices temporal resolution at the attended peripheral location in order to improve spatial resolution, which is crucial for stimulus recognition.

If emotion enhances attention allocation, the emotion-attention interaction should extend to all dimensions that are affected by attention, even if this implies a decrement in performance. It specifically predicts that valid emotion cues should have a stronger detrimental effect on performance, as compared with valid neutral cues, in a temporal resolution task (i.e., a temporal gap detection task). As in Experiment 1, we employed suprathreshold targets presented in a display without distractors or masks, which minimized target spatial uncertainty.

On top of this, the negative effect of attention on temporal resolution provides a critical test for the idea that emotion potentiates attention allocation. Any decisional account based on noise exclusion (such as the restriction of a perceptual decision to the percept arising at the task-relevant location) predicts that valid cues should always benefit performance, as compared with distributed cues, whereas in this specific case (i.e., a temporal resolution task), an attentional account predicts an opposite deficit in performance. Thus, if emotional cues enhance the detrimental effect of attentional cuing on temporal resolution, this would provide strong evidence for the idea that emotion enhances the strength of a cue's attentional response.

\section{Method}

Participants, stimuli, apparatus, and procedure Twenty observers with normal or corrected-to-normal vision participated in the experiment. The target was a circular dot $\left(1^{\circ}\right.$, $65 \mathrm{~cd} / \mathrm{m}^{2}$ ), randomly presented either left or right of fixation at $4^{\circ}$ eccentricity. On half of the trials, two consecutive dots appeared, each for $45 \mathrm{~ms}$, separated by a very brief interval (10 ms). On the remaining trials, a single dot was presented for $100 \mathrm{~ms}$ (see Fig. 1). The monitor was set to a $200-\mathrm{Hz}$ refresh-rate at $800 \times 600$ pixels. Observers indicated whether the dot contained a brief temporal gap (by pressing the " $m$ " key) or whether the dot was continuous (by pressing the " $z$ " key). All other aspects of the method and analysis were identical to those in Experiment 1.

\section{Results and discussion}

The ANOVA on $d^{\prime}$ accuracies revealed a significant main effect of attention, indicating that valid cues impaired temporal resolution, as compared with distributed cues, $F(1,19)=$ $52.42, p<.001, \eta_{\mathrm{p}}{ }^{2}=.73$ (see Fig. 2). In addition, fearful cues improved performance, as compared with neutral cues, $F(1$, 19) $=7.08, p<.02, \eta_{\mathrm{p}}{ }^{2}=.27$. As in Experiment 1 , the effects of attention and emotion interacted, $F(1,19)=45.49, p<$ $.001, \eta_{\mathrm{p}}{ }^{2}=.71$. As is shown in Fig. 3, the interaction consisted of a larger attentional deficit for fearful cues $\left(0.76 d^{\prime}\right.$ difference) than for neutral cues ( $0.25 d^{\prime}$ difference). Planned comparisons showed that the attention effect was significant for both fearful and neutral cues, $t(19)=9.37, p<.01$, and $t(19)=3.13, p<.01$, respectively (Loftus \& Masson, 1994). Also, fearful distributed cues, as compared with neutral distributed cues, improved temporal resolution, $t(19)=6.99$, $p<.01$. As predicted, these findings indicate that the emotional significance of the cue enhanced the detrimental effect of attention of temporal resolution, which is consistent with the attentional response hypothesis. As in Experiment 1, effects on criterion $C$ and RTs were not significant ( $p s>.19$ ).

\section{Correlation analyses}

To further examine the emotion $x$ attention interaction effects, we tested whether there was any systematic relationship between the magnitudes of the main effects of emotion and attention and the magnitude of the emotion $\times$ attention interaction effect for individual observers. Our reasoning was that if emotion enhances a cue's attentional response, an observers' overall attention effect should correlate with the increase in attentional effect observed for fearful versus neutral cues (see Fig. 4). We found a strong positive correlation between the attention main effect and the interaction effect (overall, $r=.86, p<.01$; spatial task only, 
$r=.76, p<.01$; temporal task only, $r=.55, p<.01$; see Fig. 4). In contrast, we found no significant correlation between the emotion main effect and the attention main effect or between the emotion main effect and the interaction effect $(p>.13)$. This indicates that the observers who showed the largest effects of attentional cuing per se also showed the largest interactions between emotion and attention.

Although there is no a priori reason to presume that participants' main effects should correlate with interaction effects within a factorial design, we wanted to test whether the correlation between the attention main effect and the emotion $x$ attention interaction was spuriously due to floor or ceiling effects. We failed to find significant correlations between the interaction effect and overall performance or between the attention main effect and overall performance ( $p$ s $>.07$ ), which excludes the possibility that individual differences in overall performance were mediating the strong correlation between the attention main effect and the emotion $\times$ attention interaction.

\section{General discussion}

A recent study by Phelps et al. (2006) demonstrated that emotion and attention have an interactive effect on contrast sensitivity: The emotional significance of a spatial cue potentiates the perceptual benefits brought about by attentional cuing. Until now, however, there was no evidence that their results extend to perceptual dimensions other than contrast sensitivity. The present study tested whether emotion enhances the effect of attention in two new dimensions: spatial and temporal resolution. In addition to extending the results of Phelps et al. to other perceptual dimensions, our design allowed us to rule out one possible explanation of the emotion-attention interaction - namely, that

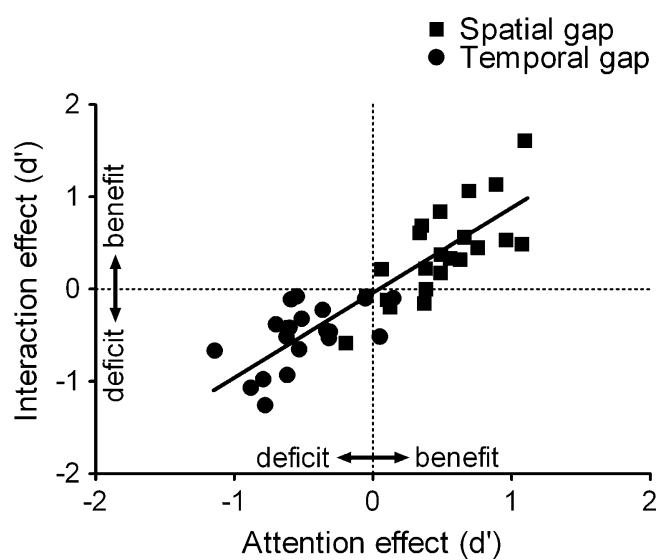

Fig. 4 Correlation between the magnitudes of the attention main effect and the magnitude of the emotion $\times$ attention interaction effect for individual observers. The attention effect was calculated by subtracting performance in the valid condition from performance in the distributed condition. The interaction effect was calculated by subtracting the attention effect for fearful cues from the attention effect for neutral cues it is due to noise exclusion (by reducing spatial uncertainty of the target location). Instead, our results show that emotion enhances the strength of a cue's attentional response.

We show that emotional cues, as compared with neutral cues, enhance the benefits of attentional cuing in a spatial gap detection task. Thus, the interaction between emotion and attention not only boosts the contrast strength of a stimulus (Phelps et al., 2006), but also improves the fine-grained spatial analysis of a stimulus (Yeshurun \& Carrasco, 1999). In addition, we show that emotional cues, as compared with neutral cues, enhance the detrimental effect of attentional cuing in a temporal gap detection task (Yeshurun \& Levy, 2003). This indicates that the emotion-attention interaction also extends to temporal properties of visual perception. It appears that emotion enhances all dimensions that are affected by attention, even if this implies a decrement in performance.

It has been proposed that emotional cues enhance the strength of a cue's attentional response at the cued location, which modulates the perceptual processing of a spatially congruent target stimulus (Phelps et al., 2006). However, spatial cues influence not only the allocation of attention, but also what subset of the presented spatial information contributes to an observer's perceptual decision. In the Phelps et al. study, observers could have had difficulty determining the spatial location of the threshold target on trials where it was presented amidst three near-threshold distractors. Thus, the more salient emotional valid cues could have helped observers to disregard distractor noise from uncued locations.

In contrast to the Phelps et al. (2006) study, our displays in Experiment 1 consisted of a single suprathreshold target in the absence of distractors and masks. Therefore, the interaction we observed between emotion and attention in spatial resolution is unlikely to have been due to a reduction in spatial uncertainty (see Carrasco et al., 2002). Also, the negative effect of attention on temporal resolution provides additional support for the idea that emotion potentiates attention allocation. Considering that decisional accounts predict that valid cues should always be advantageous, as compared with distributed cues (due to the exclusion of noise from task-irrelevant locations), the enhanced detrimental effects in temporal resolution provide strong evidence that emotion enhances attention.

We found that the magnitudes of the observers' overall attention effects in both spatial and temporal tasks were strongly correlated with the magnitude of the emotion $x$ attention interaction effect. Observers showing the largest overall attention effects also showed the largest interactions between emotion and attention, providing further support for the idea that emotion enhances the strength of a spatial cue's attentional response.

An interesting question is whether the effects observed in the present study were due to the voluntary or automatic allocation of attention (Egeth \& Yantis, 1997). Due to our use 
of a $100 \%$ predictive valid cue in combination with a multiple-element distributed cue, there was a confound between the number of cues and cue predictiveness, which prevented us from disentangling automatic and voluntary attention. Interestingly, however, it has been shown that the voluntary and automatic effects of attention can be dissociated when temporal resolution is tested in a temporal order judgment task (Hein, Rolke, \& Ulrich, 2006). In their study, exogenous cues presented peripherally at a short stimulus onset asynchrony (SOA) impaired temporal resolution, whereas endogenous cues presented centrally at a long SOA improved temporal resolution (see also Carmel, Saker, Rees, \& Lavie, 2007). This attentional dissociation in temporal resolution suggests that our short-SOA peripheral cues were engaging exogenous orienting.

Both the automatic and voluntary cuing of attention has been shown to enhance perception in the absence of target spatial uncertainty (Carrasco et al., 2002; Luck, Hillyard, Mouloua, \& Hawkins, 1996; Luck \& Thomas, 1999). Consistent with these findings, we show that attentional cues can both improve and impair the perception of a clearly localizable target (Carrasco et al., 2002; Yeshurun \& Levy, 2003). A recent study indicated that the strength of a cue's attentional response increases gradually as a function of cue contrast (Fuller et al., 2009). Consistent with this, our findings suggest that attention allocation is not a binary on-off mechanism. Rather, it appears that the strength of a cue's attentional response can vary continuously depending on both low-level (e.g., luminance contrast) and higher-level (e.g. emotional significance) stimulus parameters affecting salience.

To conclude, we have demonstrated that the interaction between emotion and attention in perception is not restricted to the contrast strength of a stimulus (Phelps et al., 2006). To our knowledge, our study is the first to extend this interaction to two new visual dimensions: spatial and temporal resolution. Combined, these findings provide strong support for the idea that emotion enhances the strength of a cue's attentional response.

Author Note Bruno R. Bocanegra and René Zeelenberg, Department of Psychology, Erasmus University Rotterdam. We thank Marisa Carrasco for helpful comments on an earlier version of the manuscript.

Open Access This article is distributed under the terms of the Creative Commons Attribution Noncommercial License which permits any noncommercial use, distribution, and reproduction in any medium, provided the original author(s) and source are credited.

\section{References}

Bocanegra, B. R., \& Zeelenberg, R. (2009a). Dissociating emotioninduced blindness and hypervision. Emotion, 9, 865-873.

Bocanegra, B. R., \& Zeelenberg, R. (2009b). Emotion improves and impairs early vision. Psychological Science, 20, 707-713.
Bocanegra, B. R., \& Zeelenberg, R. (2011). Emotion-induced trade-offs in spatiotemporal vision. Journal of Experimental Psychology: General, 140, 272-282.

Callaway, E. M. (1998). Local circuits in primary visual cortex of the macaque monkey. Annual Review of Neuroscience, 21, 47-74.

Cameron, E. L., Tai, J. C., \& Carrasco, M. (2002). Covert attention affects the psychometric function of contrast sensitivity. Vision Research, 42, 949-967.

Carmel, D., Saker, P., Rees, G., \& Lavie, N. (2007). Perceptual load modulates conscious flicker perception. Journal of Vision, 7(14), 113.

Carrasco, M., Williams, P. E., \& Yeshurun, Y. (2002). Covert attention increases spatial resolution with or without masks: Support for signal enhancement. Journal of Vision, 2, 467-479.

Carrasco, M., \& Yeshurun, Y. (2009). In N. Srinivasan (Ed.), Progress in brain research: Vol. 176. Attention (pp. 65-86). Amsterdam: Elsevier.

Chica, A. B., \& Christie, J. (2009). Spatial attention does improve temporal discrimination. Attention, Perception, \& Psychophysics, 71, 273-280.

Egeth, H. E., \& Yantis, S. (1997). Visual attention: Control, representation, and time course. Annual Review of Psychology, 48, 269-297.

Ekman, P., \& Friesen, W. (1976). Pictures of facial affect. Palo Alto, CA: Consulting Psychologists.

Ferneyhough, E., Stanley, D. A., Phelps, E. A., \& Carrasco, M. (2010). Cuing effects of faces are dependent on handedness and visual field. Psychonomic Bulletin \& Review, 17, 529-535.

Fuller, S., Park, Y., \& Carrasco, M. (2009). Cue contrast modulates the effects of exogenous attention on appearance. Vision Research, 49, 1825-1837.

Hein, E., Rolke, B., \& Ulrich, R. (2006). Visual attention and temporal discrimination: Differential effects of automatic and voluntary cueing. Visual Cognition, 13, 29-50.

Loftus, G. R., \& Masson, M. E. J. (1994). Using confidence intervals in within-subject designs. Psychonomic Bulletin \& Review, 1, 476-490.

Luck, S. J., Hillyard, S. A., Mouloua, M., \& Hawkins, H. L. (1996). Mechanisms of visual-spatial attention: Resource allocation or uncertainty reduction? Journal of Experimental Psychology: Human Perception and Performance, 22, 725-737.

Luck, S. J., \& Thomas, S. J. (1999). What variety of attention is automatically captured by peripheral cues? Perception \& Psychophysics, 61, 1424-1435.

Macmillan, N. A., \& Creelman, C. D. (1991). Detection theory: A user's guide. New York: Cambridge University Press.

Mayfrank, L., Kimmig, H., \& Fischer, B. (1987). The role of attention in the preparation of visually guided saccadic eye movements in man. In J. K. O'Regan \& A. Levy-Schoen (Eds.), Eye movements: From physiology to cognition (pp. 37-45). New York: North-Holland.

Montagna, B., Pestilli, F., \& Carrasco, M. (2009). Attention trades off spatial acuity. Vision Research, 49, 735-745.

Phelps, E. A., Ling, S., \& Carrasco, M. (2006). Emotion facilitates perception and potentiates the perceptual benefits of attention. Psychological Science, 17, 292-299.

Rolke, B., Dinkelbach, A., Hein, E., \& Ulrich, R. (2008). Does attention impair temporal discrimination? Examining nonattentional accounts. Psychological Research, 72, 49-60.

Smith, M. L., Cottrell, G. W., Gosselin, F., \& Schyns, P. G. (2005). Transmitting and decoding facial expressions. Psychological Science, 16, 184-189.

Yeshurun, Y., \& Carrasco, M. (1999). Spatial attention improves performance in spatial resolution tasks. Vision Research, 39, 293306.

Yeshurun, Y., \& Levy, L. (2003). Transient spatial attention degrades temporal resolution. Psychological Science, 14, 225-231. 